\title{
Editorial
}

\section{H1N1 (Pandemic) 2009 Influenza : Our Action Plan for Future}

\author{
KAK Azad
}

The contemporary outbreak of Pandemic H1N1 2009 Influenza, is a unique form of the old Influenza A virus. It's a triple reassortment virus meaning that it is a combination of the genetic material of classical Swine H1N1, Human H3N2 and Avian influenza viruses known as the North American Swine H3N2 and H1N2 that eventually converted into the 2009 Human H1N1 virus. Direct transformation of Classical Swine H1N1 also occurred. The entire process culminated in to a very fast human to human transmission of these influenza viruses giving rise to a pandemic form. In later part of April, 2009 the outbreak was first started in Mexico and up to the 11th of September, 2009 there were 277,607 confirmed cases throughout the world with 3,205 deaths. ${ }^{1}$ Out of this, 22,387 cases were from the SEARO (South East Asian Region) where 221 died. ${ }^{2}$ As of 7 March 2010, worldwide more than 213 countries and overseas territories or communities have reported laboratory confirmed cases of pandemic influenza H1N1 2009, including at least 16713 deaths. ${ }^{3}$

Symptoms of H1N1 influenza are generally mild. Most recover in a week, even without treatment. Some people do not realise they have it. But a small number (mostly with underlying health conditions) will develop much more serious issues. The overwhelming majority of cases have been mild and self-limiting, with no need for treatment. Cases of severe or fatal infections have been largely, but not exclusively, confined to people with underlying chronic conditions. First case was identified in Bangladesh on 18th June, 2009. The number of lab confirmed cases in Bangladesh stood at 372 with death of 3 cases till 13th of September, 20094 and up to 28th February cumulative confirmed cases were 827 and among them 6 were died. ${ }^{3}$ According to latest update of H1N1 2009 pandemic by WHO on 12 March 2010, in Bangladesh, overall influenza activity remains low although an increasing trend in respiratory disease was reported. ${ }^{3}$

Currently lab confirmation is no longer required in Bangladesh as well. 'Flu Corner' and 'Flu Ward' were made fully operational in the tertiary care hospitals of the country along with Bangabandhu Sheikh Mujib Medical University (BSMMU), Dhaka Medical college Hospital and a number of private hospitals in Dhaka. ${ }^{5}$ Because this is a new ("novel") virus, most people will not have immunity to it and so illness may be more severe and widespread as a result. The further the virus spreads, the more chance it will mix, or "reassort" with other flu viruses in circulation and turn into something more lethal. April to September is the Influenza season in Bangladesh; IEDCR and ICDDRB are working on seasonal influenza and according to Institute of Epidemiology Disease Control \& Research (IEDCR) the predominant flu strain active in this season of 2009 was the H3N24. Recently a guideline for management of Pandemic Influenza H1N1 - 2009 in Bangladesh has been formulated with technical assistance from the Bangladesh Society of Medicine and made available which states the do's and don'ts along with categorization of cases into category A, B and $C$, definition of risk group and appropriate management protocols and clear indication for the use of antiviral therapy. For Category A - home self Isolation and social distancing till symptoms (about 7 days) abate, supportive care, paracetamol, antihistamine, respiratory etiquette(cover mouth and nose with mask, if a face mask is not available tissue/ handkerchief/piece of clothe/'sari anchol' /'gamcha' / shirt's sleeve/scarf or even elbow may be used to cover cough and sneeze, to practice spitting at defined places, to avoid touching the eyes, nose, and mouth, frequent hand washing with soap and water etc), but no antiviral (Oseltamivir) is recommended. For Category B - above measures plus antiviral (Oseltamivir) is recommended. For Category $\mathrm{C}$ - above measures for category B plus hospital admission (HDU or ICU if needed) is recommended. Risk Group includes Infants and children ( $<5$ years), elderly ( $>65$ years), pregnant women, patients with chronic co-morbid conditions such as cardiovascular, renal, respiratory or liver disease, diabetes mellitus, those with

KAK Azad, Professor, Department of Medicine, Dhaka Medical College Hospital, Dhaka-1000, Bangladesh, E-mail : markazad24@yahoo.com 
immunosuppression related to malignancy, chemoradiotherapy, organ transplant recipient, HIV infection etc \& obesity defined as a BMI $>30.6$ This guideline made things easier for the treating physicians regarding decision making. A well formulated preventive strategy has also been undertaken by the Government of Bangladesh. Health support desk was opened in 15 ports along with international airport in Dhak ${ }^{5}$. All secondary and tertiary level hospitals have been provided with adequate storage of Oseltamivir and trained staff to combat the emergency. All district hospitals made complete isolation unit by the end of the 2009. The Government has adequate stock of Oseltamivir. ${ }^{4}$

The closure of schools and educational institutes is a controversial issue. The WHO has no proper guideline regarding when to take such a decision. Rather they have left the whole matter for the local authorities to decide. The pros and cons are described in their guideline. ${ }^{7}$ In such a densely populated country like Bangladesh, the success of limiting contact from school absenteeism is quite questionable.

According to the WHO this is only the first wave of Pandemic Influenza H1N1 - 2009, the second wave is yet to come. The only measure to prevent this is to vaccinate people. United States Food and Drug Administration (US-FDA) has approved an effective vaccine on the 15th of September, 2009 and provided manufacturing license to four companies. ${ }^{8}$ Initially it was thought that two shots will be required for effective immunization. China first came up with the proof that in fact a single shot is good enough. ${ }^{9}$ United States of America in conjunction with Australia, Brazil, France, Italy, New Zealand, Norway, Switzerland and United Kingdom has announced the donation of pandemic vaccine to the developing nations. ${ }^{10}$ So it is important for us to formulate a strategy regarding vaccine for its sooner availability and regarding vaccination by identifying priority groups based on susceptibility. This will ensure proper utilization of the limited amount of vaccine that we will receive.

Although we had been able to control our part of this pandemic quite satisfactorily so far and the number of cases and casualties were well within the tolerable range, to ensure that things remain as good as it is now or even better than that we have to keep up the vigilance and modify the measures well in time to overcome new situations. ${ }^{5}$

Last of all we have to remember that we are a poor nation with limited resources and technical support and we have such a land that is world's most densely populated. So any inconvenience regarding handling this issue may cause catastrophy and an unbearable damage to the nation if we are not adequately prepared for action plan to combat the situation that may arise in near future.

\section{References}

1. World Health Organization. Situation report-Pandemic H1N1 2009 (13 September, 2009). www.whoban.org/ pdf/ SitRep_A_(H1N1).pdf Accessed September 24, 2009.

2. World Health Organization. Summary of Situation of Pandemic H1N1 2009 for week of 7 - 13 September 2009. Available at: www.searo.who.int/EN/ Section10/ Section2562_15050.htm Accessed September 24, 2009.

3. WHO | Pandemic (H1N1) 2009 - update 91

4. Institute of Epidemiology, Disease Control and Research. Influenza A H1N1 (Swine Flu) Fact Sheet (updated on 16- 092009). Available at: www.iedcr.org Accessed September 24, 2009.

5. Editorial, Pandemic (H1N1) 2009 Influenza:Bangladesh Perspective, H AM Nazmul Ahasan, J Bangladesh Coll Phys Surg 2009;27:129-131

6. Institute of Epidemiology, Disease Control and Research. Treatment Guideline for Influenza A H1N1 (updated on 19- 082009). Available at: www.iedcr.org/images/PDF/ Treatment_guideline.pdf Accessed September 24, 2009.

7. World Health Organization. Measures in school settings. Available at: www.who.int/csr/disease/ swineflu/notes/ h1n1_school_measures_20090911/en/index.html Accessed September 24, 2009.

8. Neergaard L. FDA Approves Swine Flu Vaccine. Associated Press. Available at: news.aol.com/health/article/fdaapprovesswine- flu-vaccine/446911 Accessed September 24, 2009.

9. Schmid RE, Cheng M. China approves single-dose swine flu vaccine.Associated Press.Available at: www.physorg.com/ news171178203.html Accessed September 24, 2009.

10. World Health Organization. Pandemic vaccine donations for the developing world. Available at: www.who.int 